\title{
The Significance of the Logo of NEOM in Plasticizing Contemporary Abstract Artworks
}

\author{
Elham Abdullah Rayes ${ }^{1}$ \\ ${ }^{1}$ Department of Art Education, College of Education, Umm Al-Qura University, Saudi Arabia \\ Correspondence: Elham Abdullah Rayes, Department of Art Education, College of Education, Umm Al-Qura \\ University, Saudi Arabia. E-mail: elham.rayes@gmail.com
}

Received: February 28, 2018

Accepted: March 30, 2018

Online Published: June 28, 2018

doi:10.5539/ies.v11n7p54

URL: https://doi.org/10.5539/ies.v11n7p54

\begin{abstract}
The current study aimed at plasticizing contemporary artworks by activating the formal and denotative significances of the logo of NEOM through the concept of abstract art. To achieve the study objectives, participants were asked to plasticize contemporary artworks using multiple-sized canvas, several coloring materials, a computer and some software as a technological medium, and high-quality printer papers to activate the significances of the logo of NEOM. Abstract artworks were displayed and analyzed in the light of activating the formal and denotative significances of the logo of NEOM. The author follows the descriptive approach and the quasi-experimental approach. The experiment was applied to female students in the Arts Lab at Umm Al-Qura University in 2018/ 1439H. The experiment yielded the ability of activating the logo of NEOM in artworks (plastic works), combining the significances of the logo of NEOM and abstract art produces unusual artworks, plastically activating the logo of NEOM resulted in enriching the aesthetic value of artworks, and formal and denotative significances of the logo of NEOM enrich plastic arts especially abstract works. The author recommends keeping up with contemporary progress and events and inspiring plastic artworks from the reality around the artist.
\end{abstract}

Keywords: logo, NEOM, contemporary, abstract artworks

\section{Introduction}

Art is an aesthetic human condition that developed and varied throughout history ranging from the art of primitive man, through the arts of different civilizations including the Pharaonic, Phoenician and Greek to the modern and postmodern arts (Miller et al., 2009).

Modern art is characterized by the removal of barriers among its various forms. It also calls for integrating the different forms and materials under "visual arts" category (AlBahnasi, 1982).

Art plays a significant role in our vision of environment and nature, which is not possible unless art shows them to us. As a result, we recognize their beauty in the language of expression. Artistic expression is the expression of meanings in the language of form, color, word or movement. Each mass has a meaning, each gesture has significance, each color has a reference, and each word has an image. Art is always related to the surrounding circumstances whether they are social, economic...etc. Social history of art asserts that artworks do not only express individual awareness, they also reflect the social attitudes towards the world (Attiah, 1994).

In KSA, the track of Vision 2030 is increasingly shaped. It is particularly based on transforming Saudi Arabia from oil-based economy to depending on various resources. Moreover, it aims to ease the burden on the state due to the backward economy and to broaden and support the private sector in order to create more jobs for the Saudi youth.

Designing the logo of NEOM is regarded a way of visual communication that depends on a concise idea or message in order to publicize the significances of the logo. It develops access to the outside world to express the cognitive significances and convey many meanings through the language of form, line and color. It is associated with the characteristics of abstract art whether it is the geometric abstraction or abstract expressionism through symbols inspired by areas to be implemented within the plan of the project. The logo is based on an outer geometric drawing and an inner expressive drawing. All of them clearly show the significances to be communicated to the receiver in order to understand the idea of the project.

According to this vision, the present study activates the formative and denotative significances of the logo of 
NEOM in plasticizing contemporary artworks depending on geometric and expressive bases. Each part of the logo has a denotative significance: The trees indicate the environment, the fingerprint indicates arts, the blue squares indicate energy, the interlaced circles indicate communication, and the digital network indicates technology (arabic.cnn.com).

They all combine the formal beauty and the denotative significance serving art education, plastic arts, and plastic photography, in particular.

\subsection{Statement of the Problem}

Thus, the problem of the study can be summarized in asking the following major question: Can the significances of the logo of NEOM be employed to generate new visions of plasticizing contemporary artworks?

\subsection{Objective}

The study aims at plasticizing contemporary artworks by activating the formal and denotative significances of the logo of NEOM through the concept of abstract art.

\subsection{Significance}

The significance of the study can be presented as follows:

1) Keeping up with the current development in KSA by plastically activating the significances of the logo of NEOM.

2) Tackling a vital branch of contemporary plastic art, i.e. abstract art.

3) Highlighting the role of formal and denotative significances of the logo of NEOM in enriching plastic art.

\subsection{Limitations}

Limitations of the study are categorized as follows:

\subsubsection{Objective Limitations}

1) Abstract art and its techniques.

2) Formal and denotative significances of the logo of NEOM and semantics.

3) Producing contemporary abstract artworks.

4) Literature review.

\subsubsection{Temporal Limitations}

The present study is conducted in $2018(1439 \mathrm{H})$.

\subsubsection{Spatial Limitations}

The experiment was applied to female students in the Arts Lab at Umm Al-Qura University.

\section{Method}

The study adopts the descriptive, analytical and quasi-experimental approaches.

- The descriptive approach is a method of describing the topic to be studied through a valid scientific methodology. Then, results are expressed as digits that can be interpreted (Obaydat et al., 1999).

- $\quad$ The analytical approach is used to study reality or a phenomenon. It accurately describes and expresses it quantitatively and quantitatively (Obaydat, 2004).

- $\quad$ The quasi-experimental approach depends on studying the human phenomena as they are, or it is based on studying the relation between two variables without controlling them.

\section{Literature Review}

The following sections review some relevant studies:

Gerges (2013) aims at designing commemorative stamps expressing the most important stages of the Palestinian cause in order to completely express those stages by unifying formal and plastic formulas, color stamp, type of writing, and sizes in all of the implemented commemorative stamps. The study adopts the descriptive analytical and experimental methods. It concludes that here is lack of interest in the Palestinian stamp that completely expresses all the aspects of the Palestinian cause and identity. Accordingly, it recommends that Palestinian commemorative stamps have to include the most important active and significant symbols to successfully communicate the stamps' advertising messages. Besides, those symbols shall be artistically and aesthetically employed in terms of the basics of stamp designing. The present study agrees with Gerges's in employing symbols 
and significances. They differ in the source of data and the field to be enriched by employing those significances. Qasemy (2005) aims at identifying the significances of place in expressionistic stage presentations with specific focus on the plays of the College of Fine Arts. The study adopts the descriptive analytical method in describing the significances of place and analyzing expressionistic stage presentations. It agrees with the present study in being based on defining the concept of significances and adopting the same method. However, while the present study explores the significances of the logo in the field of plastic arts, Qasemy (2005) proves to be useful for the present study in that it comprehensively defines the concept of significances and denotative expressionistic meanings .

Ghamdi (2004) is an attempt to identify the educational significances derived from the verses of patience in the doctrinal, moral and ethical aspects and identifying their educational applications to family and school. The study adopts the deductive method. It agrees with the present study in clearly defining the concept of significances, but the present study differs in searching for the formal and denotative significances of the logo, while Ghamdi's adopts a deductive method special for the significances of the Holy Qur'an. It helps the present study in identifying the significances and denotative values that can be deduced from the logo of NEOM.

\section{Definition of Terms}

\subsection{Significances}

Significance is what signifies something. It refers to a formative intentional meaning aiming at stimulating subjective and objective meanings and analyzing them into facts related to the receiver's mind in order to participate in activating the relation between the signifier and the signified (AlKasmi, 2005).

It is procedurally defined as what leads to recognizing something as the significance of formal and denotative symbols and images in designing the logo of NEOM.

\section{$4.2 \operatorname{Logo}$}

It is a "graphic mark, emblem, or symbol used to aid and promote public recognition. It may be of an abstract or figurative design or include the text of the name it represents, including a country, city, union of countries or organizations, institution, company or body (wikipedia.org).

\subsection{NEOM}

It is a Saudi project of a planned transnational city. It is part of the ambitious aspirations of Vision 2030 aiming at transforming KSA into a global pioneer model in all aspects of life (wikipedia.org).

\subsection{The Logo of NEOM}

It is a logo designed as a pentagram; each side has a symbolic drawing related to the vision of NEOM (Al-Fifi, 2017).

It is procedurally defined as an image symbolizing five different aspects of life that are aimed to be developed by the project; each aspect has its own formal and denotative significance.

\subsection{Plasticizing}

Linguistically, plastic arts are types of drawing or sculpturing which involve drawing natural and social views and so on in specific sizes and figures according to their drawings (Almaany Arabic Dictionary).

They refer to the practical application of scientific theories using appropriate means. They are acquired by studying and creating things that enjoy the mind through feelings (Dakheel, 2008).

Procedurally, they express a language that an artist tries to convey by a new artistic idea that distinguishes him. They also refer to plasticizing contemporary abstract artworks based on employing the significances of the logo of NEOM.

\subsection{Abstract Artworks}

Abstract art is the philosophical framework where abstracting is based on the essential features of natural forms by abstracting aesthetic traits of sensual forms and images (Hassan, 2003).

Procedurally defined, abstract artworks are based on transcending the familiar forms towards more free artworks depending on pure relations between the plasticizing elements that may be geometric or expressive that expresses the intention of the artist.

\subsection{Contemporary}

The term describes something living or occurring at the same time or following modern ideas in style or design (Pearsall, 2002). Procedurally, it refers to the contemporary creative artwork related to the artist's ability to keep up 
with the developments, changes and events of his present time.

\section{Theoretical Framework}

\subsection{Abstract Art and its Techniques (1910)}

Exploring plastic arts in previous times reveals the emergence of new features of freedom of expression and respect for the artists' subjectivity. Some artworks call for revolting against traditions of the past in order to cope with the zeitgeist through creativity. Those artists combined artistic schools; each one had its own artistic vision and doctrine. For example, there were the New Classicism, Romanticism, Realism, Impressionism, Expressionism, Fauvism, Cubism, and Abstract Surrealism (Azzam, 1999).

Abstractism was introduced in 1910 aiming to achieve abstract creativity of simulation. In other words, it aims to express abstracting pure forms of their sensible details, which do not imply any real features. It also aims to get artistic results through form, line and color (Azzam, 199). The first pure abstract artwork was created by the Russian painter and art theorist, Wassily Kandinsky.

The major theory of abstract art is based on materialism. It appeared for the first time in nearly 1915 in the works of the Russian structuralist artists who paid great attention to the essence, forms, colors and patterns, and rejected the technique of narration, poetry and emotional experiences. They positively formed the new era and its scientific pillars.

The term was initially used at the end of World War II in 1945 referring to the art that is totally free from material or subject of the drawing. Then, pure abstraction got widely famous due to the works of abstract expressionists and the artists of New York School such as Jackson Pollock, Arshile Gorky and Franz Kline.

Abstract art employs symbols to refer to metaphysical elements in order to reach out to absoluteness. It transforms natural forms from their occasional shapes into immortal essential forms; i.e. moving from partial properties to total qualities (Hassan, 2003). It describes the process of abstracting the essence of a natural form to be presented in a new one. Since the era of Cézanne, abstract artists have been far from imitating nature. Then, Cubists followed them by disjoining natural forms to be reformulated by a new geometric technique (AbuDibsah and Gaith, 2008). Thus, sceneries are transformed into just triangles, squares and circles. Then, the abstract painting appears as accumulated scraps of paper, sectors of rock or clouds, i.e. it appears as just harmonized pieces that do not have direct visual significances even if it implies some aspects of the artist's plastic experience. Generally, abstractism intends to find out and express the essence of things as concise forms bearing the artistic experience that nudged the artist's imagination. The word "abstraction" denotes the elimination of all the traces of reality (Yahia, 1993).

\subsection{Techniques of Abstract Art}

\subsubsection{Abstract Expressionism}

It is also called abstract art. Abstract Expressionism moved away from the traditional ideas of plastic values and basic features through extreme emotion characterized by humanitarian aspects such as the artist's subjective vision of himself demolishing structural visual aspects to be replaced with his own world to be painted (Yahia, 1993).

Its most prominent artist was Wassily Kandinsky who was the leader of this movement. His abstract works went through several stages and could be divided into three periods, as follows:

a) When he lived in Germany, it was characterized by abstract designs of expressionistic forms.

b) When he moved away from the previous expressionistic stage and his abstraction took on a geometric character, he depended on coherent design, not on colors. His paintings were characterized by integrated geometrical designs full of straight lines and arcs. That was clear in his Multicolored Circle (Figure 1).

c) It was the result of his previous experiences when he lived in Paris (1933-1944). It was distinguished by a non-objectives technique characterized by coherent design and harmonized lines. That was portrayed in his Transverse Line, 1923 (Figure 2). 


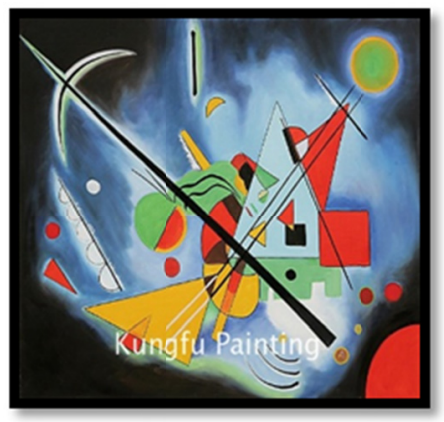

Figure 1. Multicolored Circle by Wassily Kandinsky, 1921, Yale University Art Gallery, USA

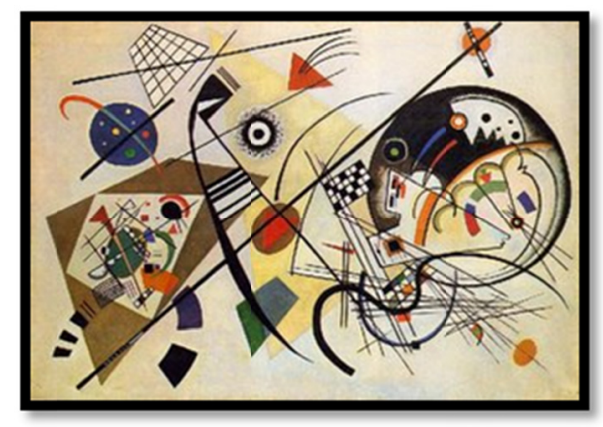

Figure 2. Transverse Line, 1923 by Wassily Kandinsky, The Solomon R. Guggenheim Museum, New York

Mikhail Larionov (1881-1964): He was one of the most prominent abstract artists in Russia. He created a new technique in abstract art named as "Rayonism" and his works were of popular primitive character. That was clear in his Autumn 1912 (Figure 3).

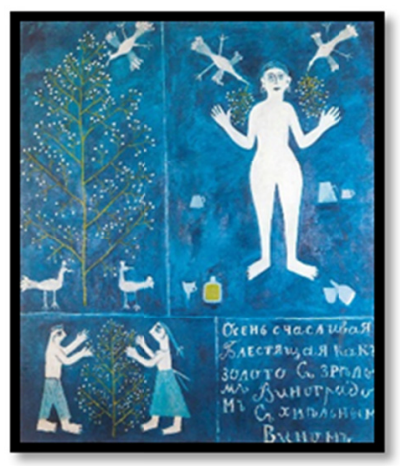

Figure 3. Mikhail Larionov, Autumn 1912, Kunstmuseum Basel, Switzerland

\subsubsection{Geometric Abstraction}

It is also called non-objective art. Its artists believe that the mission of art does not only depend on reformulating things in nature, but on expressing them through absolute facts. They depend on vertical and horizontal lines and basic colors starting from structural abstraction passed by geometricism to geometric approximation and gradual reduction.

The most famous artists of the movement were Mondrian (Figure 4), (Zmary, 2014), Malevich (Figure 5), Delaunay and Albers (Allam, 2010). 


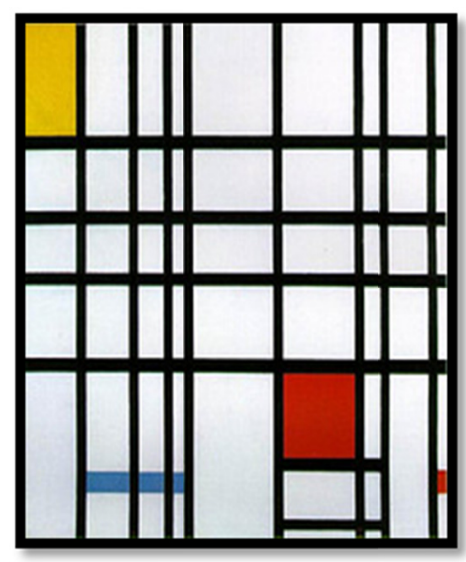

Figure 4. Piet Mondrian, composition with blue, red and yellow 1921, Oil on canvas, Tate Modern, London

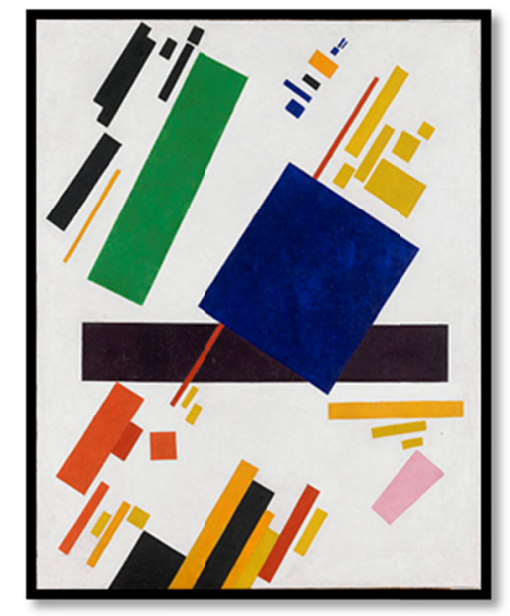

Figure 5. Kazimir Malevich, Suprematist, Oil on canvas, 1878-1935

\subsection{Formal and Denotative Significances of the Logo of NEOM and Semantics}

\subsubsection{Identity and Components of the Logo}

A logo is not only a figure, icon or decorated name, it also expresses the philosophy of the services and activities provided by the company so that it is entrenched in minds. It expresses the trust between consumers and the company or service producer. Thus, once one sees the logo of a famous company, s/he trusts the service that the company provides (wikipedia.org).

A logo is usually designed to quickly convey an idea, to enable people to easily identify its owner without getting confused with similar companies and to visually distinguish something. It is often combined of two elements: a picture or symbol, and text written in distinctive calligraphy. Sometimes, it is a registered trademark. Therefore, a logo can be so simple that its simplicity is the secret of its beauty (wikipedia.org).

\subsubsection{Characteristics of a Logo}

1) Distinctive, i.e. cannot be easily confused with other logos.

2) Practical, i.e. can be used as is in different fields.

3) Can be clearly used in small or large sizes.

4) When printed, it keeps its general shape and characteristics (wikipedia.org).

\subsubsection{Criteria of Designing a Logo}

- Distinctiveness: A logo primarily aims at distinguishing its company. So, it has to be distinctive and unique 
in order not to resemble any other logos.

- Simplicity: A logo has necessarily to be simply understood. It has not to be exaggerating in employing colors and symbols or it would be complicated and difficult to understand (Gaber, 2016).

- $\quad$ Maximizing and minimizing: A professional logo stays clear when maximized or minimized.

- Clarity: A logo has to stay clear even it is printed in black and white because it is supposed to be officially used in correspondence among institutions, countries and companies (Gaber, 2016).

\subsection{Semantics}

Semantics is an old branch of knowledge that combines authenticity and contemporary. Anees (1984) explains the consensus that language is originally derived from sensual things. He argued that authors agree that semantics started with senses and it evolved to abstract indications as the human brain evolved. The more thought is evolved, the more it tends to extract and generate abstract indications to be used later.

\subsubsection{Types of Semantics}

a) Lexical Semantics: It refers to the unity of the denoted meaning. In other words, each word has a central meaning or fixed name in the external atmosphere and it denotes something in life referring to it.

b) Metaphorical Semantics: It refers to words conveying other meanings and it can be called denotative semantics.

c) Contextual/ Artistic Semantics: It is the significance of word within its context and structure because words relate to each other which would lend them a new expressive value (AlDaya, 1996).

\subsection{Formal and Denotative Significances of the Logo of NEOM:}

The project of NEOM represents a new future and history. It will be the most appropriate place for living all over the world to absorb the future talents (The Saudi Press Agency http://www.spa.gov.sa). It will be constructed on a 26,500 $\mathrm{km} 2$ active special zone located in the northwest of the Kingdom. On the west and north, it overlooks the Red Sea and the Gulf of Aqaba on $468 \mathrm{~km} 2$ of coastline. On the east, it is surrounded by mountains up to 2,500 meters high. It will be a better place for the best mindsets and companies to reach the highest levels of invention (Figure 6). The great project will connect three continents together due to its strategic location that is considered a meeting point connecting the Arab Region, Asia, Africa, Europe and America (AlNahar Newspaper, https://www.annahar.com).

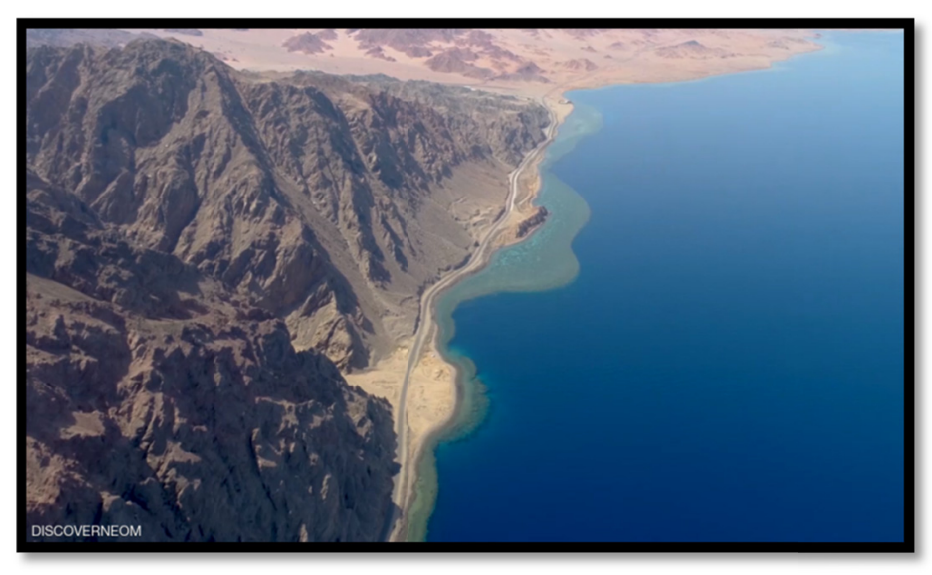

Figure 6. The region of the project of NEOM

The project presents global standards of living for individuals (Figure 7). Those standards include cultural, artistic and educational sides, advanced urban planning, high-quality living style, and civil and technological services of health, education, transportation and entertainment, in addition to (Almadaen Online Newspaper). 


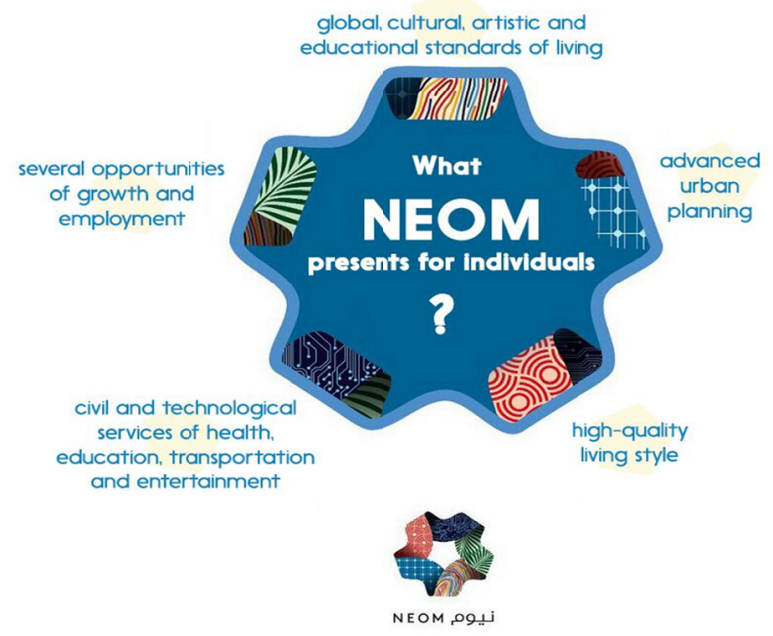

Figure 7. The global standards of the logo of NEOM

NEOM project aims at achieving several objectives:

1- For individuals: Building a global community with the brightest future talents that embrace innovation and creativity and show respect for all nations.

2- For prosperity: providing the best environment for investors and innovators to build the future economic model that promotes cooperation for sustainable development.

3- For the planet: redefining the most suitable lifestyle in this unique destination to promote sustainable future as well as healthy and balanced lifestyle

In addition to its economic role, resources and prospects, the logo of NEOM, with its various colors and five sides, reflects several aspects significances of the project.

\subsection{Denotative Significances of the Logo of NEOM (Interpreting the Name):}

The word NEOM is divided into two parts (Figure 8): The first three letters form the Greek prefix neo- meaning "new". The fourth letter is from the abbreviation of "Mostaqbal; an Arabic word meaning "future. Thus, NEOM means the new future. Overall, the word NEOM denotes a word referring to the future. It also implies something from the outer space (Sabq Online Newspaper).

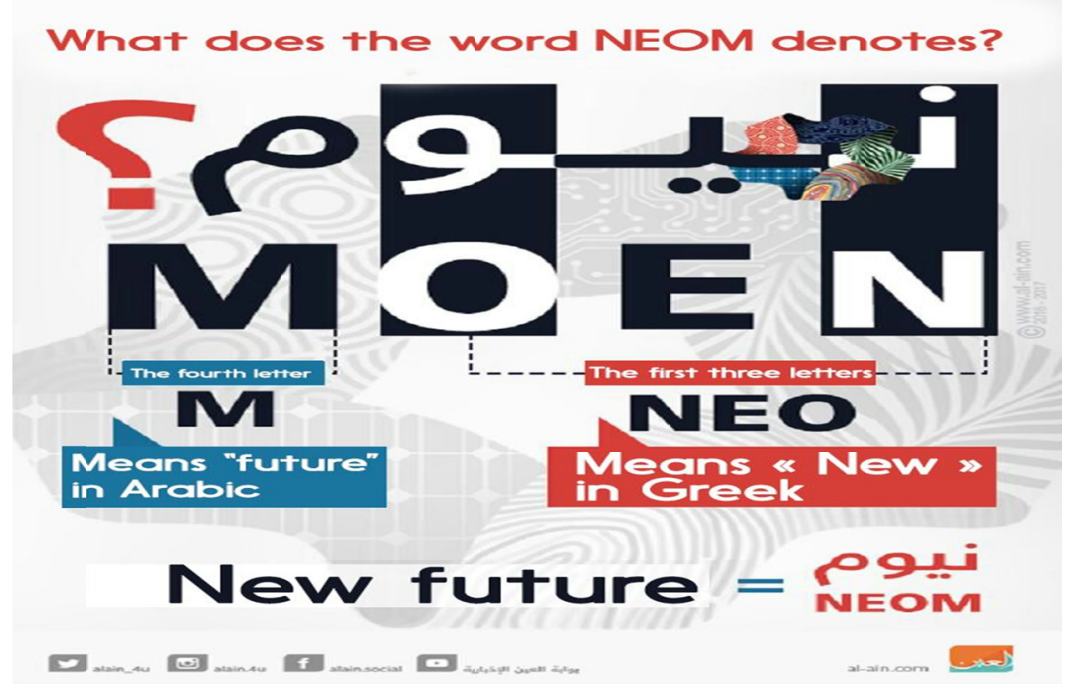

Figure 8. Denotative significances of the logo of NEOM 


\subsection{Formal Significances of the Logo of NEOM:}

It is a logo designed as a pentagram; each side has a symbolic drawing related to the vision of NEOM. This vision indicates that NEOM will be the city where anyone in the future dreams of living in (Figure 9).

In addition, each figure has significance. While trees indicate the environment, the fingerprint indicates arts, the blue squares indicate energy, the interlaced circles indicate communication, and the digital network indicates technology (arabic.cnn.com).

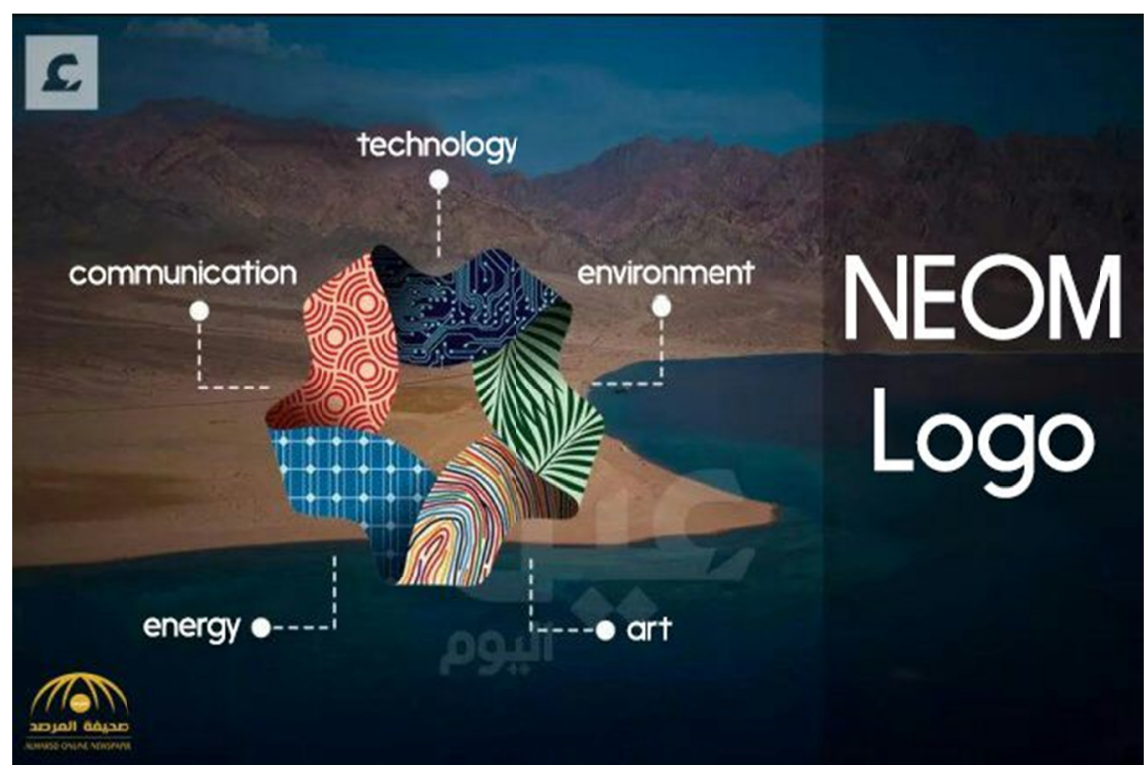

Figure 9. Formal significances of the logo of NEOM

- The first side represents the world where the environment is protected and dealt with as one element of life on planet.

- The second side symbolizes technology and programming which are the top of today's international investments leading the fourth industrial revolution towards the future.

- The third side refers to the communication and cultural missions of NEOM presented as interwoven decorations indicating the pioneer role of the city in supporting the cultural track as part of the ideal world.

- The fourth side is designed as a solar cell indicating the project's great investment aimed at developing clean energy and depending on it as a primary source of energy in the city.

- The fifth side is a colored fingerprint because the project offers a civilized mission of openness to the world's different identities, cultures and colors regardless of the small exclusive identities that endeavor to remove any chance for hybridization between cultures. Hence, the city is symbolized as a place for all human beings to achieve their dreams.

Those five sides of the logo of NEOM are formal significances interpreting the meanings that reflect the idea of the project. Human beings try to achieve that idea for it holds their dreams true (CNN Arabic).

An expressive inspiration of the analysis of the significances of NEOM's logo

The logo of NEOM indicates the Islamic star (Figure 10); it is consisted of:

- Palm trees $=$ Arab

- $\quad$ Fingerprint= human beings

- $\quad$ Electronic panel= technology and evolution

- $\quad$ Network $=$ system

- $\quad$ Circles $=$ embracing all cultures (Figure 11) 


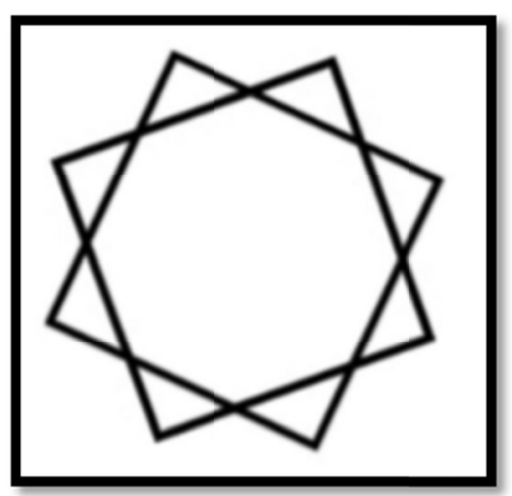

Figure 10.

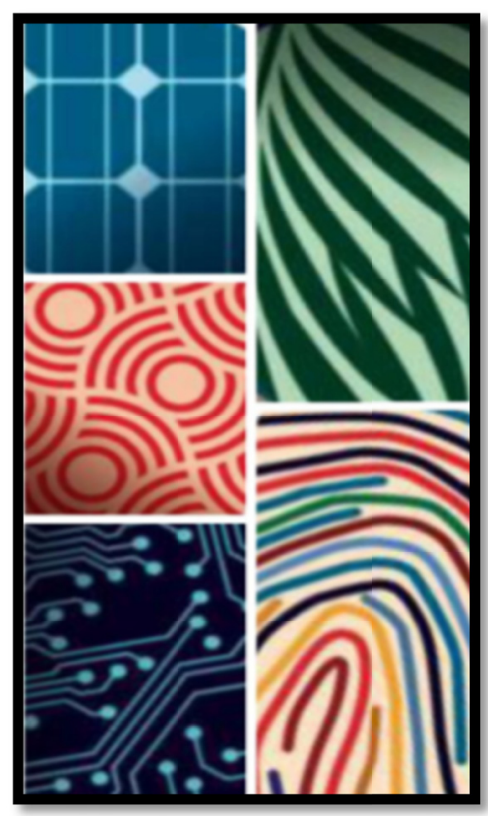

Figure 11.

We all have dreams, but we do not have the same vision. While some people look to their dreams with closed eyes and they cannot see the way for achieving them, others perceive their dreams with wide-open eyes and strong resolution to achieve them. The logo of NEOM is about "dream" that will inevitably lead to prosperity and progress. Out of the principle of dream, the logo of NEOM is employed in abstract plastic artworks to cultivate the imagination of female students in the production of their artworks.

\subsection{The Relation of NEOM's Logo to Abstract Art}

The design of NEOM's logo is one of the ways of visual contact that depend on conveying the idea or message in order to publicize the significances of the logo of NEOM.

The design of NEOM's logo develops communication with the outside world to express the cognitive significances and convey many meanings through the language of line and color. It is associated with the characteristics of abstract art whether it is a geometric abstraction or abstract expressionism through symbols inspired by the areas to be implemented within the plan of the project. Each area is symbolized by abstracting its expressive elements on each side of the logo of NEOM. The logo is based on an outside geometric drawing and inside expressive drawing. They all clearly show the significances to be communicated to the receiver in order to understand the idea of the project.

\section{Applied Experiment}

Based on the theoretical framework, the author tends to do the practical experiment in order to answer the major question of the study: Can the significances of the logo of (NEOM) be employed to generate new visions to plasticize contemporary artworks?

\subsection{Population and Sampling}

The population of the study includes female students enrolled in the course of Color Expression No. (234212) in the second level of the final year, Department of Art Education, College of Education, Umm Al-Qura University, Mecca.

\subsection{Objectives of the Experiment}

1) Employing the significances of the logo of NEOM according to a scientific research about producing contemporary plastic artworks.

2) Providing students with new experiences in producing contemporary plastic artworks based on employing the significances of the logo of NEOM.

3) Highlighting a vital branch of contemporary plastic art, i.e. abstract art and expressing it by producing 
contemporary plastic artworks.

\subsection{The Artistic Aspect of the Experiment}

On employing the formal and denotative significances of the logo of NEOM depending on abstract attitude, a contemporary plastic artwork is produced.

\subsection{Objects/Tools Used in Implementing the Experiment}

Multiple-sized canvas, several coloring materials, a computer and some software as a technological medium, and high-quality printer papers.

The description and analysis of the plastic artworks produced by students are presented as follows:

Artwork 1: (Figure 12). My home is my environment

Pastel dry and soft colors are applied to a rectangular canvas. The composition depends on that the vertical line is perpendicular to the horizontal line achieving balance. Also, the Arabic word "watany" meaning "my home" is drawn in order to reinforce the concept of home. The material used for the tableau is a cloth decorated by some popular decorations of the art of "Asiri Katt" (a Saudi traditional art). The significances of the logo of NEOM inspired by the first side (environment and nature) are employed in this artwork. The first side of the logo is reduced and abstractly symbolized by green trees in addition to strong yellow and orange stains referring to the color of the sun that would enlighten the country promising a new bright future. Colored squares are scattered indicating the logo of Vision 2030 that encompasses the project of NEOM. The digits 2030 were written at the bottom of the painting evoking the continuous presence of Vision 2030.

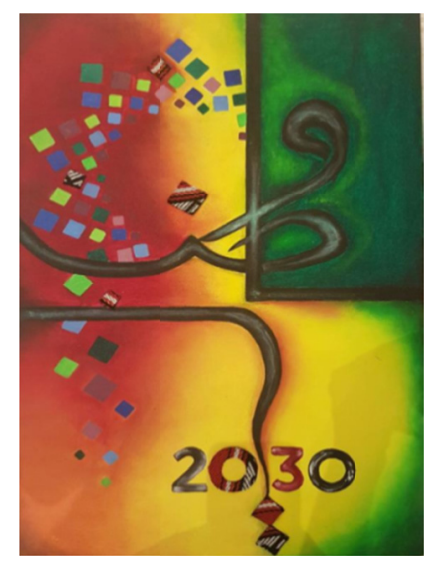

Figure 12. My home is my environment

Artwork 2: (Figure 13). The dialogue of vision

Dry Pastel colors are softly applied embodying the faces of King Salman and his Crown Prince face to face as if they were talking about the Vision in order to assure their role in launching Vision 2030 encompassing the project of NEOM. The painting shows some expressive meanings revealing that they think of the country's concerns and wish for a better future. Geometric abstraction is highlighted through faces and spaces on the background. The significance of the logo of NEOM appears when employing the fifth side (colored fingerprint) in order to confirm the cultural message that reveals the meaning of openness to different kinds of world identities and cultures. 


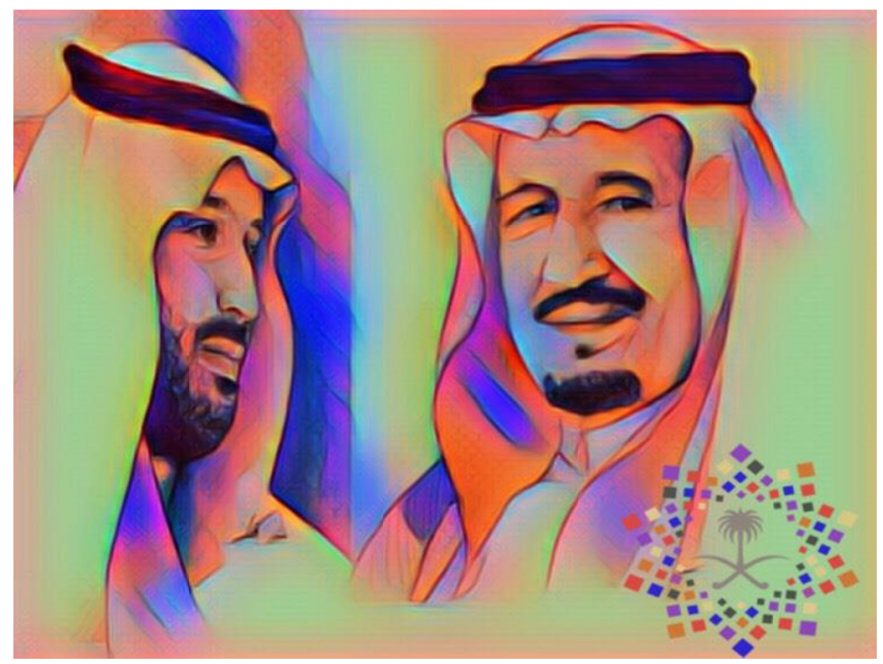

Figure 13. The dialogue of vision

Artwork 3: (Figure 14). Towards the Future

It is drawn using Pastel colors and the technique of Air Brush in an abstract style. The composition is an ambiguous white spot, shining as a flash amidst a dark background - that we can think of as a human face. Also, the background is intervened with red and blue spots spirally scattered around the figure. There are white vertical lines emerging from the ambiguous figure; each line ends with a blue dot as if they are leading people to the future. Finally, the significances of the logo of NEOM are employed to express technology and programming which constitute the second side of the logo.

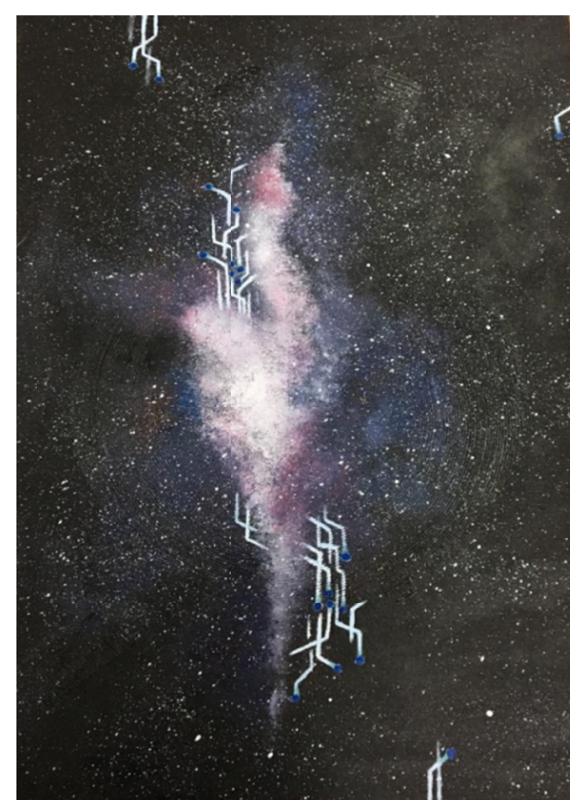

Figure 14. Towards the future

\section{Artwork4: (Figure 15). Embrace}

This artwork is made of oil colors, acrylic colors and gold leaves on a background of Gesso by rough and soft touches of gradual blue. The interweave between the abstract expressionistic style and the geometric abstract style is presented through the two hands embracing each other amidst the background. Around the two hands, there are linear rhythms of semi-circles which are basically forming the left hand. The semi-circles are intervened with straight refracted white lines which are basically forming the right hand. Therefore, the significances of the second and third sides of NEOM's logo were employed, referring to the overlap between technology, programming and 
communication. All of those elements are embodied as interwoven decorations indicating the pioneer role of the city in supporting the cultural track as part of the ideal world.

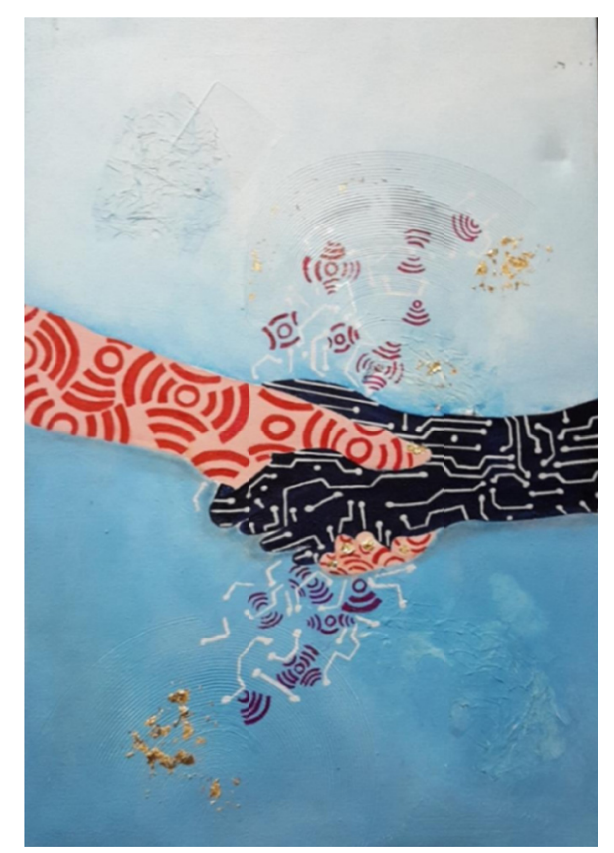

Figure 15. Embrace

Artwork 5: (Figure 16). Revolution

It is drawn using acrylic and pastel colors on a background of Gesso. The composition is based on a central point in the middle where there are refracted beam lines emerging from the middle and extending to the end of the painting. Those lines look like a strong flash of lightning striking in the middle of a very dark sky which evokes that the future revolution will be that of programming and technology to be at the top of global investments leading to the fourth industrial revolution towards the future.

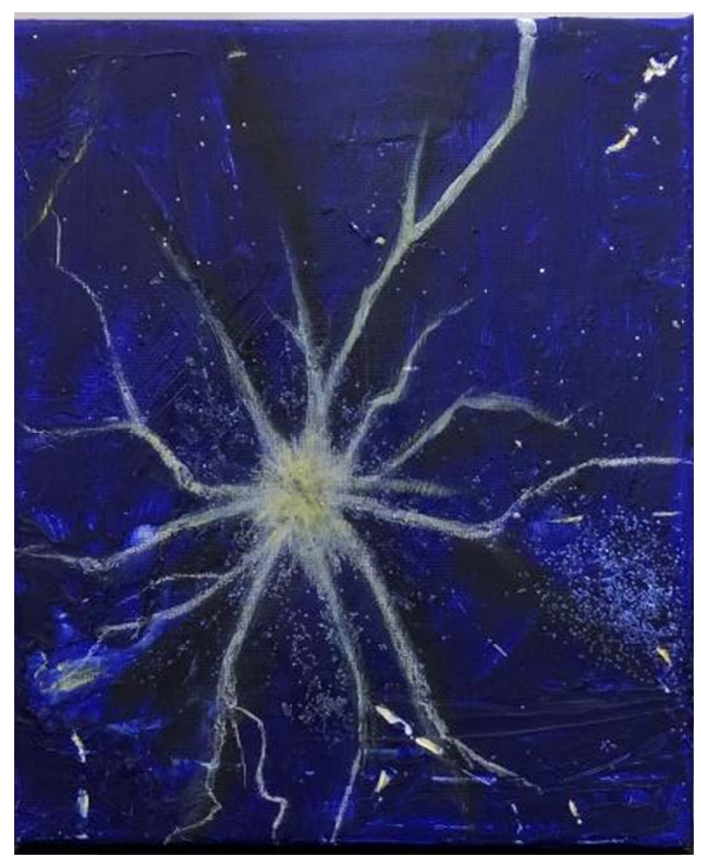

Figure 16. Revolution 
Artwork 6: (Figure 17). Like father, like son

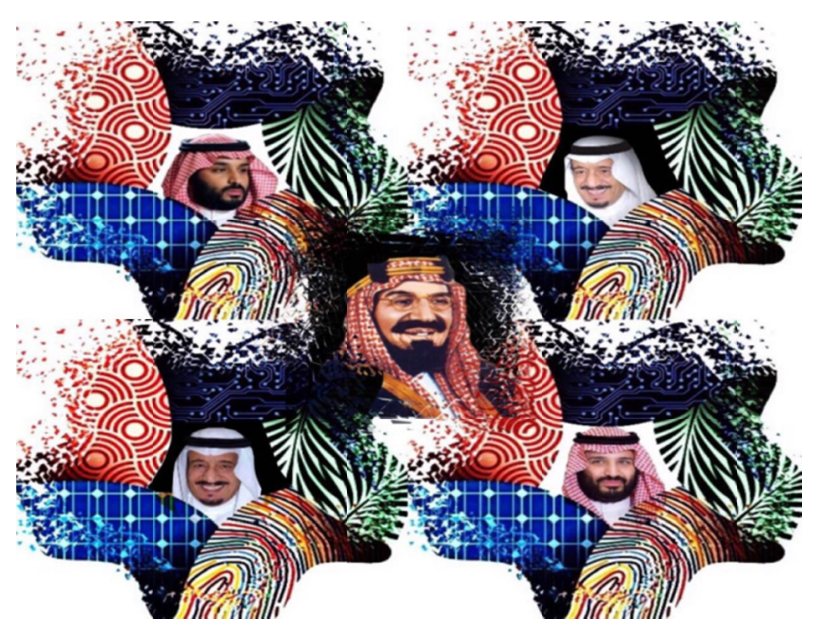

Figure 17. Like father, like son

Digital technology was employed in the artwork using (Adobe Photoshop) which is Pixel Art software. The artist employed the structural style by abstractly combining more than one photo by repeating the item of NEOM's logo which is designed by diverse abstract decorations. The logo is surrounding the photos of pioneer leaders whose insightful visions express successive generations and visions evoking the proverb: "Like father, like son". It is here that the formal and denotative significances of the logo of NEOM with all its five sides appear.

Artwork 7: (Figure 18). Building human beings

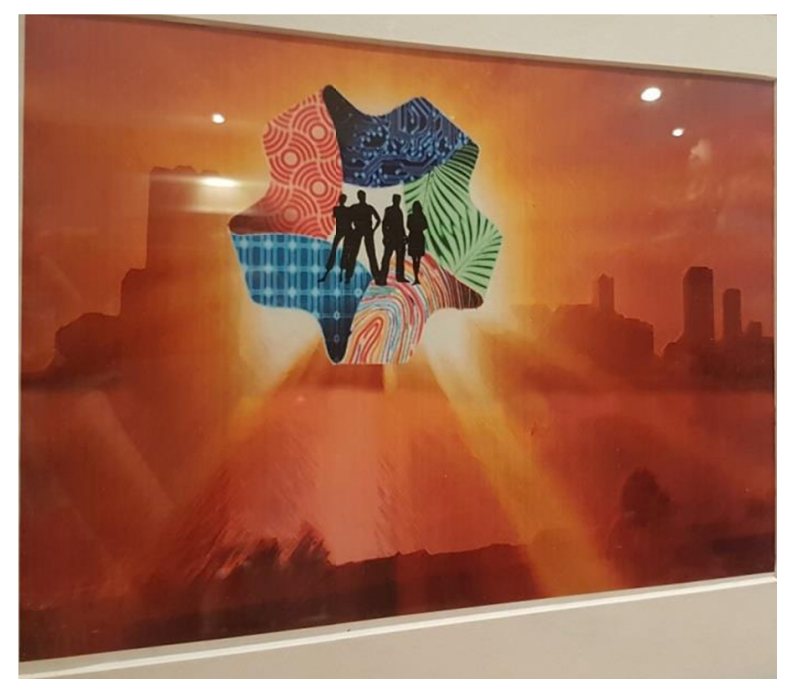

Figure 18. Building human beings

Digital technology was employed in this artwork using (Adobe Photoshop). The technique employed here is that of incorporating several photos. The logo of NEOM appears exactly at the middle of the composition heading up and carrying inside it photos of some people symbolizing the human beings: a father, mother, son, family, employee, worker...etc. Each one plays one's roles in this life. People have to cooperate in order to achieve human progress and improvement. 


\section{Results}

In the light of the displayed plastic artworks, for example but not limited to, the study concluded some results. The formal and denotative significances of the logo of NEOM can be utilized in plasticizing as well as analyzing plastic artworks created by the participants. The experiment yielded that combining the significances of the logo of NEOM and abstract art produces unusual artworks. In addition, Plastically activating the logo of NEOM resulted in enriching the aesthetic value of artworks. Formal and denotative significances of the logo of NEOM enrich plastic arts based on abstract thinking. Contemporary abstract artworks created by the participants contribute to educating the members of the society about how the formal and denotative significances of the logo of NEOM can be utilized in creating artworks with future style and high aesthetic value. The significance of the logo of NEOM is of valuable important, as helped participants create contemporary artworks and led to the expansion of their imagination.

\section{Recommendations}

In the light of the concluded results, the study recommends:

1) Adopting and supporting the trend towards employing the formal and denotative significances of the logo of NEOM in creating plastic artworks.

2) Directing the attention of artists to the importance of employing the formal and denotative significances of the logo of NEOM in creating contemporary plastic artworks.

3) Issuing specialized artistic journals to highlight the importance of activating the formal and denotative significances of the logo of NEOM in creating plastic artworks, to spread the culture of the significances of the logo as well as to publish it on various websites.

4) Conducting more studies on the project of NEOM and its denotative significances.

5) Keeping up with contemporary progress and events, as well as inspiring plastic artworks from the reality around the world.

\section{References}

AlBahnasi, A. (1982). The art of Europe from the Renaissance up till now. Lebanon: Al Raed Al Arabi.

AlDaya, F. (1996). Arabic semantics: Theory and practice. Damascus: Dar Al Fikr Publishing House.

Al-Fifi, Z. (2017). The story behind NEOM logo. Retrieved from https://goo.gl/F5y3fB. Acceded on 20/11/2017

Allam, N. (2010). The arts of the West in modern times. Egypt: Dar Almaref

Anees, I. (1984). The significance of words. Egypt: The Anglo - Egyptian Bookshop.

Attiah, M. (1994). Art and social life. Egypt: Dar Almaref.

Azzam, A. (1999). Art appreciation and criticism in plastic arts. KSA: AlMofradat for Publishing and Distribution.

Dakheel, A. (2008). Plastic art exhibitions between academic preparation and practical application. Department of Art Education, College of Education, King Saud University.

Gaber, A. (2016). The concept of logo. Retrieved from http://mawdoo3.com/\%D9\%85\%D9\%81\%D9\%87\%D9\%88\%D9\%85_\%D8\%A7\%D9\%84\%D8\%B4\%D8 $\% \mathrm{~B} 9 \% \mathrm{D} 8 \% \mathrm{~A} 7 \% \mathrm{D} 8 \% \mathrm{~B} 1$

Gerges, S. (2013). Utilizing Palestinian national symbols in the design of postage stamps (MA thesis, Helwan University, Egypt).

Ghamdi, N. (2004). Educational significances derived from the verses of the Holy Qur'an and their applications to family and school. Umm Al-Qura University, Saudi Arabia.

Hassan, M. (2003). Trends of contemporary art: A detailed explanation of all modern artistic trends. Giza: Hala for Publishing and Distribution.

Miller, B. L., Cummings, J., Mishkin, F., Boone, K., Prince, F., Ponton, M., \& Cotman, C. (1998) Emergence of artistic talent in frontotemporal dementia. Neurology, 51, 978-981. https://doi.org/10.1212/WNL.51.4.978

Obaydat, M., Abu Nassarah, M., \& Mobaydeen, O. (1999). Scientific research methodology: Rules, stages and applications. Amman: The University of Jordan.

Obaydat, Z., Adas, A. R., \& Abdel Haq, K. (2004). Scientific Research: Techniques and Tools. Magdalawy for 
Publication and Distribution.

Pearsal, J. (2002). Oxford English dictionary. Oxford: Oxford University Press.

Qasemy, S. (2005). The significances of place in expressionistic stage presentations (M.A. thesis, University of Babylon, Iraq).

Yahia, M. (1993). Pre-and-post expressionism plastic values. Egypt: Dar Elmaref.

Zmary, M. (2014). What is graphics? Retrieved from http://mawdoo3.com /\%D9\%85\%D8\%A7 \%D9\%87\%D9\%88_\%D9\%81\%D9\%86_\%D8\%A7\%D9\%84\%D8\%AC\%D8\%B1\%D 8\%A7\%D9\%81\%D9\%8A\%D9\%83. Acceded on 18/11/2017

\section{Appendix}

In the following section, the author presents some more artworks as an appendix about the applied experiment: Figures $19(1,2,3,4,5,6)$.

(1) Stability

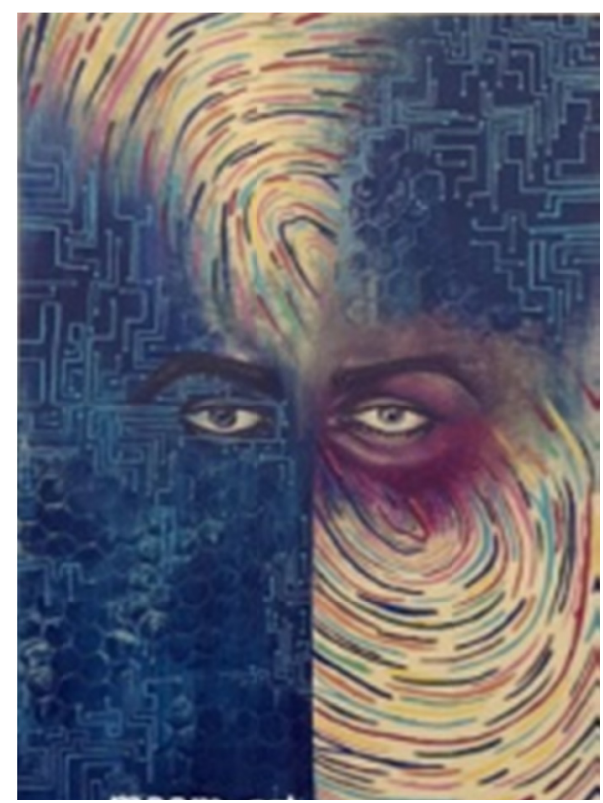

(3) Progress

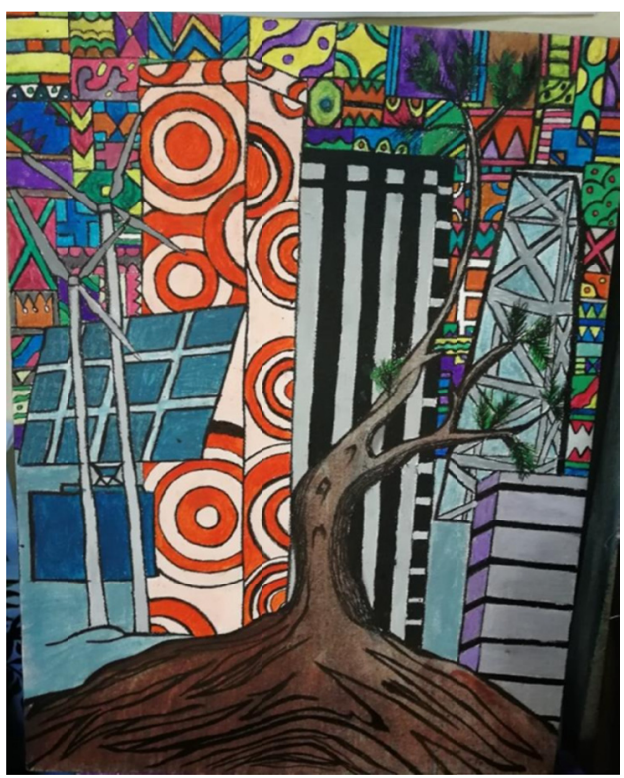

(2) Vision

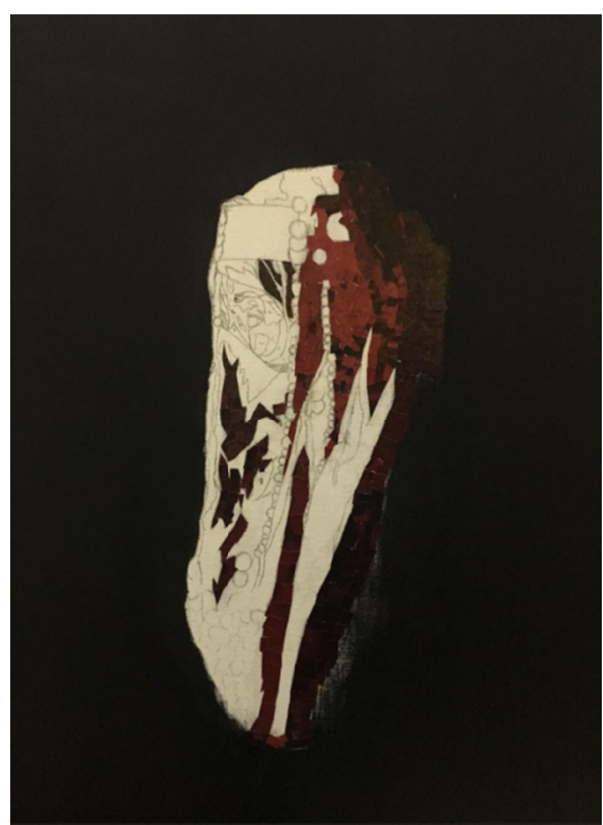

(4) National Doctrine

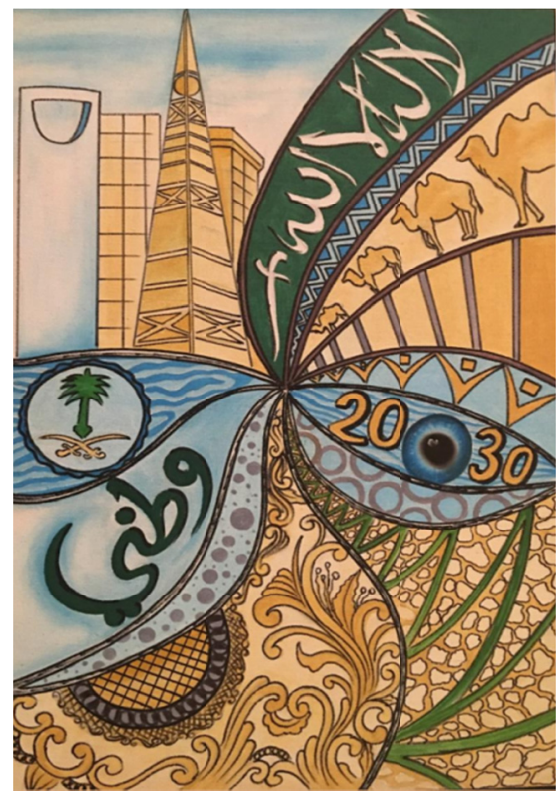


(5) Insightful Gaze

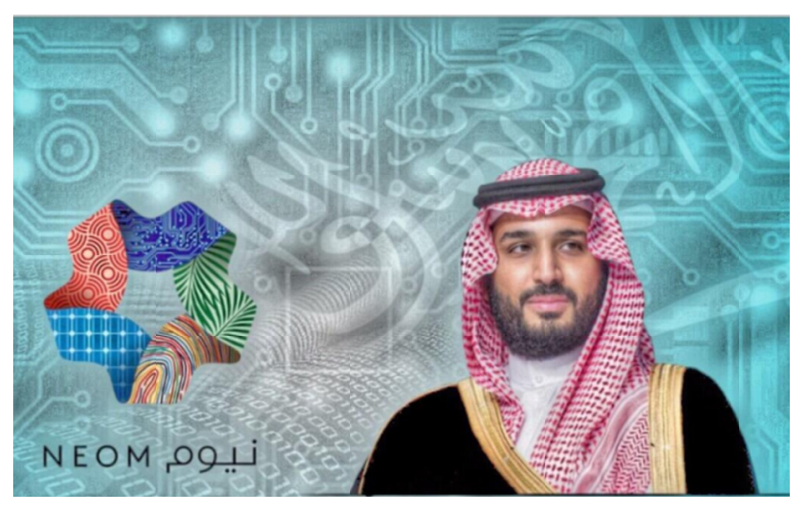

(6) Space

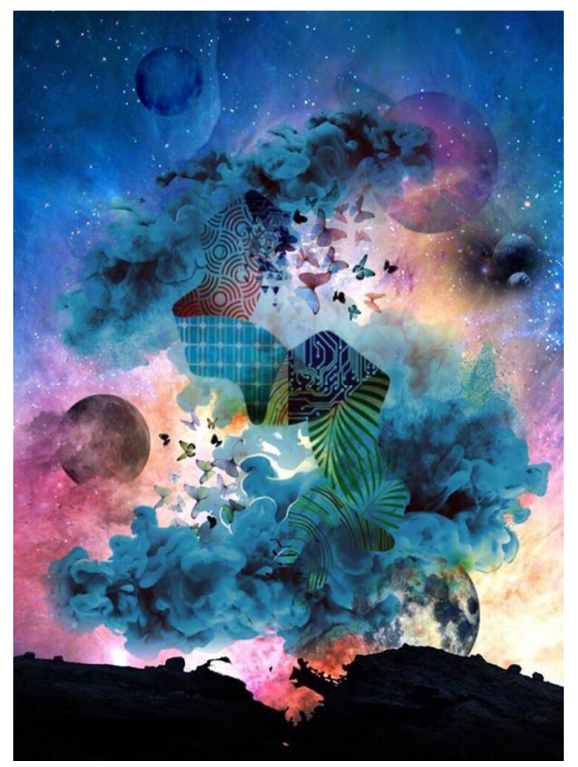

\section{Copyrights}

Copyright for this article is retained by the author(s), with first publication rights granted to the journal. This is an open-access article distributed under the terms and conditions of the Creative Commons Attribution license (http://creativecommons.org/licenses/by/4.0/). 